\title{
Pedagogía de la convivencia y educación no formal: un estudio desde el ocio físico-deportivo, los valores y la familia
}

\author{
Ma Ángeles Valdemoros, Ana Ponce de León, Rafael Ramos y Eva Sanz \\ Universidad de La Rioja (España)
}

El ser humano se concibe en una constante interrelación con variados agentes educativos de los que fluyen diversos valores y actitudes. El presente estudio pretende verificar la idoneidad del ocio físico-deportivo para el fomento de valores sociales dirigidos a optimizar la sociabilidad y la convivencia de los adolescentes considerándose, asimismo, a la familia como agente cardinal de este proceso. Se ha explorado la percepción que jóvenes, padres y profesores tienen sobre la dimensión social de los valores en el ámbito de análisis. Para tal fin se ha utilizado una complementariedad metodológica trabajando con técnicas cuantitativas -empleando como instrumento de medida un cuestionario- y con técnicas cualitativas -caso del análisis de contenido de cuatro grupos de discusión-. Los resultados ratifican que el ocio físico-deportivo es, a priori, un contexto axiológico neutro, que posee el potencial de fomentar tanto valores como contravalores; que la familia se constituye en un agente educativo fundamental susceptible de condicionar el hábito físicodeportivo del adolescente; por último, a pesar de que los padres defienden una práctica promotora de valores sociales, los profesores y los jóvenes les reprochan el hecho de no valorarla en su justa medida facilitándose, de este modo, el abandono de dicho estilo de vida.

Palabras clave: Educación no formal, convivencia, ocio físico-deportivo, familia, adolescentes.

The pedagogy of living together and nonformal education: a study based on physical-sport leisure, its values, and the family unit. A human being conceives of him or herself as being in a constant interrelation with varied educative agents, through which diverse values and attitudes flow. The present study seeks to verify the suitability of the physical-sport leisure model to promote social values that optimize an adolescent's ability to integrate and socialize, while considering the family unit to be the cardinal agent in this process. The scope of the analysis, therefore, explores the perceptions of young people, parents, and professors regarding the social dimensions of these values. To this end, a complementary methodology has been used that works with quantitative techniques -employing a questionnaire as an instrument of measurement- and with qualitative techniques, specifically a contextual analysis of four discussion groups. The results confirm the following: that a physical-sport leisure model is, a priori, a neutral axiological context, with the potential to encourage as many values as counter-values; that the family unit constitutes both an educative and fundamental agent that is able to engender physical-sport habits in the adolescent; and that in spite of parents defending the practice of social values, teachers and young people sometimes reproach them for not valuing it in just measures, potentially leading to the neglect of the above lifestyle.

Key words: No formal education, living together, physical-sport leisure, family, adolescents.

Correspondencia: Ana Ponce de León Elizondo. Universidad de La Rioja. C/ Luis de Ulloa, s/n. 26004. Logroño (España). E-mail: ana.ponce@ unirioja.es 
El ser humano es un ente individual y social. La persona no se forma sola, sino que se inserta en una constante interrelación con variados agentes educativos, sociales, políticos, culturales, religiosos, morales, etc. de los que fluyen, igualmente, diversos valores, actitudes y pautas de comportamiento que interactúan con cada uno de nosotros desde que nacemos y que nos van configurando como seres únicos e irrepetibles.

Asimismo, tenemos una necesidad de vinculación y comunicación; cuestiones que, a priori, resultan beneficiosas porque implican compartir metas comunes y sentir que los demás pueden ser fuente de ayuda y satisfacción, entre otros aspectos; sin embargo, también son susceptibles de conflictividad, dado que surge el enfrentamiento entre los deseos e intereses propios y los de los otros (Marina, 2006).

La sociabilidad es nuestra capacidad para relacionarnos con los demás y supone tanto una aptitud cuanto una actitud para la convivencia que, por otro lado, no es igual en todas las personas. Para ello, el papel de la acción educativa es fundamental. Según los planteamientos de la UNESCO en el Informe Delors (1996) la respuesta pedagógica ha de construirse sobre cuatro pilares fundamentales, siendo uno de ellos el de aprender a vivir juntos.

Coincidimos con Jiménez (2005) en la aseveración de que a convivir se aprende. Se trata de una acción que exige tolerancia, así como compromiso con las normas instauradas con el fin de serenar los conflictos y favorecer el bienestar integral.

La educación se constituye en un espacio vital de transmisión de conocimientos, capacidades, valores, actitudes, destrezas, así como normas de comportamiento susceptibles de posibilitar que la persona alcance una armonía entre su felicidad individual y social. De acuerdo con Kant (1983), la educación es un arte cuya práctica debe perfeccionarse y renovarse a través de las generaciones con el fin de que la persona alcance su meta; en el caso que nos ocupa, la de aprender a convivir de forma saludable. En esta empresa están implicados numerosos agentes educativos que intervienen desde diversos ámbitos de actuación.

Dado que este estudio se centra en el marco de uno de los espacios de la educación no formal, el que compete a las experiencias de ocio físico-deportivo (OFD), así como en la influencia de la familia como agente fundamental condicionante en este contexto, se considera fundamental reflexionar sobre el marco axiológico deseado para nuestros adolescentes en la esfera que nos ocupa, pues en función de la educación en valores que se establezca para los mismos así se predice el modelo de persona que anhelamos para nuestra sociedad (López-Pastor, 2003) y, por ende, el paradigma de convivencia al que aspiramos.

Llegados a este punto, nos parece oportuno poner de manifiesto la descripción que Marina (2006) establece para definir una familia inteligente, declarando que es aquella que implanta lazos de afecto, de colaboración y de comunicación que hacen sentirse a todos sus miembros más seguros y felices, de la que surgen unas normas de vida, unos valores sociales y una ética rectora del comportamiento, entre otras cuestiones. De ahí que la familia se constituya en un agente cardinal para la educación axiológica. 
En este sentido resulta fundamental que el núcleo familiar eduque y, análogamente, socialice; es decir, que ayude a instaurar en sus hijos el modelo de persona que nuestra sociedad expresa como óptimo. En primer lugar, ha de revisar y meditar sus capacidades, valores, actitudes y normas de convivencia para, de este modo, garantizar la transmisión de una sociabilidad adecuada a nuestros niños y jóvenes, los cuales serán, en definitiva, quienes avalen un futuro mejor para nuestra convivencia ciudadana.

En síntesis, el quehacer educativo puede y debe llevarse a cabo desde los ámbitos formales, no formales e informales de la educación; ámbitos, todos ellos, fundamentales. En el presente artículo vamos a centrarnos en uno de los contextos de educación no formal, el de las experiencias de ocio físico-deportivo; contexto, a nuestro parecer, fundamental por su potencialidad en la transmisión de valores y prevención de contravalores. Asimismo, conscientes de que son muchos los agentes educativos y sociales implicados en esta tarea, caso de la familia, los docentes, los monitores de tiempo libre y el grupo de iguales, entre otros, focalizaremos nuestra atención en la familia por considerarla pieza clave en el engranaje de la educación en valores.

A continuación, se estima necesario realizar una revisión del estado de la cuestión en referencia al tema objeto de estudio, comenzando por aquellas investigaciones, tanto del ámbito nacional como internacional, que indagan en el potencial de las experiencias de ocio físico-deportivo para el fomento de una convivencia saludable.

Se considera oportuno iniciar este apartado señalando que, bajo nuestro punto de vista, el ocio físico-deportivo, como ámbito de educación no formal, se constituye en un contexto axiológico neutro capaz de desarrollar tanto valores como contravalores, dependiendo del enfoque educativo que se lleve a cabo. Dicha percepción coincide con la de otros investigadores del ámbito que nos ocupa (Borrás, 2004; García-Ferrando, 1990; Giebink y McKencie, 1985; Lee y Cook, 1990).

Queda ampliamente constatado en diferentes investigaciones que en estas experiencias la búsqueda de relaciones sociales goza de alta consideración entre los adolescentes, quienes destacan la amistad como motivo importante de inicio y mantenimiento en su práctica físico-deportiva (Castillo y Balaguer, 2001; Griffin, 1985; Moreno y Hellín, 2002; Ponce de León, Sanz, Valdemoros y Ramos, 2009; Valdemoros, 2010; Velázquez y Hernández, 2003).

Recíprocamente, queda igualmente demostrado el papel fundamental de dicha práctica en el fomento de la integración social, de las habilidades y competencias, así como en la prevención de algunos efectos del aislamiento social (Pelegrín, 2004; Raga, 2002; Reitman, O'Callaghan y Mitchell, 2005; Valdemoros, 2010).

En otro orden de cosas se estima, de acuerdo con otros autores (Hassandra, Goudas y Chroni, 2002; Moreno y Cervelló, 2004; Nuviala, Ruiz-Juan, García-Montes, 2003; Vilhjalmsson y Thorlindsson, 1998), que la familia es pieza clave entre los agentes educativos implicados en las experiencias de ocio físico-deportivo de niños, adolescentes y jóvenes en cuanto a su influencia en la motivación y situación de práctica de los mismos, constatándose un aumento de la probabilidad de adherencia y continuidad en este tipo de prácticas si la familia posee un estilo de vida activo. 
Asimismo, queda verificado que el núcleo familiar ejerce enorme influencia en la transmisión de valores, actitudes y pautas de conducta. Yuste y Pérez (2008) declaran que, según la percepción de los padres, uno de los factores con mayor poder de influencia en el origen de las conductas violentas de los adolescentes es la ausencia de educación en valores en la familia, destacando la falta de respeto como contravalor fundamental. Se considera que esta cuestión es susceptible de trasladarse al ámbito del ocio físico-deportivo; aspecto que avala el hecho de que la educación axiológica en el ámbito familiar pueda constituirse en un factor preventivo de los contravalores, también en la práctica físico-deportiva (PFD).

No obstante, es alentador constatar que padres y madres defienden una práctica físico-deportiva promotora de valores, así como que destacan la necesidad de fomentar un trabajo actitudinal que evite comportamientos agresivos y optimicen el desarrollo integral del adolescente (Edo, 2004; Kremer-Sadlik y Kim, 2007; Ramos, Sanz, Ponce de León y Valdemoros, 2009; Vizcarra, Macazaga y Rekalde, 2006).

Como fruto de la justificación presentada se intuye que el ocio físicodeportivo, como ámbito de educación no formal, posee el potencial de constituirse en el espacio eficaz para llevar a cabo una pedagogía de la convivencia que fomente aquellos valores que optimizan el bienestar social del adolescente constituyéndose, en definitiva, en un contexto favorecedor para el aprendizaje de la socialización.

Asimismo se prevé que la familia, estimada como agente educativo fundamental implicado, puede llegar a ejercer una gran influencia tanto en el inicio y mantenimiento de dichas prácticas como en la no adhesión y abandono de las mismas, así como en la educación en valores sociales susceptibles de contribuir tanto a una convivencia saludable como al fomento de actitudes generadoras de conductas disruptivas, capaces de traspasar la frontera de la esfera familiar.

Es por ello por lo que la pretensión del presente artículo es la de analizar la importancia que conceden los padres, los profesores y los propios jóvenes a los contextos de ocio físico-deportivo como espacios susceptibles de favorecer el fomento de valores sociales que configuran el mapa axiológico de dichas experiencias, tales como la amistad y la convivencia saludable.

Además, se pretende conocer cuáles son los valores que los padres, los profesores y los jóvenes objeto de estudio establecen como idóneos para las experiencias de OFD, así como los antivalores que determinan deben ser anulados, con el propósito de comprobar si todos los colectivos caminan en una misma dirección.

Asimismo, se aspira a analizar la influencia de la familia en las experiencias de OFD de sus hijos, tanto a nivel de inicio y mantenimiento como de abandono de las mismas, procurando determinar los contravalores susceptibles de generarse en el ámbito familiar que condicionan negativamente dicho hábito.

Por último, se pretende determinar las discrepancias perceptivas entre los diferentes colectivos analizados, referentes a la influencia que ejerce la familia en la práctica físico-deportiva del adolescente, con el propósito de concentrar esfuerzos y establecer líneas coherentes de actuación entre todos los agentes educativos implicados, dirigidos a optimizar dicho estilo de vida. 


\section{METODO}

En el presente estudio se ha efectuado una triangulación metodológica, utilizando técnicas cuantitativas y cualitativas en dos fases.

\section{Fase cuantitativa:}

En una primera fase se pretende conocer si los adolescentes perciben que su familia ha influido en su historia físico-deportiva, así como analizar la importancia que los adolescentes otorgan a dichas experiencias como herramienta favorecedora de las relaciones sociales.

\section{Participantes}

El universo de trabajo de esta fase estuvo compuesto por todos los adolescentes que cursaban alguno de los cuatro niveles de Educación Secundaria Obligatoria en los centros educativos de la Comunidad Autónoma de La Rioja, éste ascendía a un total de 11.259 sujetos, cifra recogida del Informe de Estadística Escolar de la Comunidad Autónoma de La Rioja. La muestra estuvo constituida por 1.978 sujetos, valor obtenido para un nivel de confianza del $95 \%$.

\section{Instrumentos}

Se han analizado un total de seis variables: la percepción de los adolescentes sobre la importancia concedida por sus padres a la práctica físico-deportiva de sus hijos, la situación de práctica físico-deportiva actual y anterior, la situación de práctica del padre y la situación de práctica de la madre, los motivos de inicio físico-deportivos y los beneficios obtenidos de la práctica.

En la tabla 1 se detallan las variables del estudio con sus categorías, así como los ítems del cuestionario que recogen información sobre ellas.

Tabla 1. Variables, categorías e ítems

\begin{tabular}{ll}
\hline \multicolumn{1}{c}{ VARIABLES } & \multicolumn{1}{c}{ CATEGORÍAS } \\
\hline $\begin{array}{l}\text { Percepción de los adolescentes sobre la } \\
\text { importancia concedida por sus padres a la } \\
\text { práctica físico-deportiva de sus hijos. }\end{array}$ & $\begin{array}{l}\text { Ninguna importancia / Poca importancia / Bastante importancia / Cada vez más } \\
\text { importancia / Mucha importancia }\end{array}$ \\
\hline $\begin{array}{l}\text { Situación de práctica físico-deportiva actual y } \\
\text { anterior. }\end{array}$ & $\begin{array}{l}\text { No practico ni practicaba / No practico pero antes practicaba / Practico pero antes } \\
\text { no practicaba / Practico y practicaba }\end{array}$ \\
\hline Situación de práctica del padre & $\begin{array}{l}\text { No practico ni practicaba / No practico pero antes practicaba / Practico pero antes } \\
\text { no practicaba / Practico y practicaba }\end{array}$ \\
\hline Situación de práctica de la madre & $\begin{array}{l}\text { No practico ni practicaba / No practico pero antes practicaba / Practico pero antes } \\
\text { no practicaba / Practico y practicaba }\end{array}$ \\
\hline Motivos de inicio físico-deportivos & $\begin{array}{l}\text { Motivos de forma física / Motivos de gusto por la actividad / Motivos relacionados } \\
\text { con la tarea / Motivos sociales / Motivos estéticos / Motivos relacionados con el } \\
\text { ego / Motivos de salud / Motivos relacionados con la oferta / Motivos familiares / } \\
\text { Motivos temporales / Motivos educativos. }\end{array}$ \\
\hline 22 & $\begin{array}{l}\text { Mejora de salud / Forma física / Mejora estética / Gusto por la actividad / } \\
\text { Beneficios sociales / Mejora del estado psicológico / Beneficios orientados al ego / } \\
\text { Valores implícitos / Mejora de hábitos / Beneficios orientados a la tarea / } \\
\text { Ocupación del tiempo libre / Pocos o ninguno. }\end{array}$ \\
\hline 3eneficios obtenidos de la práctica & 34
\end{tabular}

El instrumento utilizado en esta fase ha sido el cuestionario MACOFYD (Ponce de León, Sanz, Ramos y Valdemoros, 2010). En su validación se empleó la 
validez de contenido (juicio crítico de expertos y test preliminar) y la de constructo (método de depuración de la información obtenida y análisis de contenido). Para la obtención de la fiabilidad del instrumento empleado se optó por calcular el coeficiente alfa de Cronbach y se obtuvo un valor 0.69, por lo que se puede afirmar la fiabilidad de la variable ante lo que se pretendía medir.

\section{Análisis de datos}

Con los datos registrados en el cuestionario se ha realizado un análisis descriptivo de cada una de las seis variables, así como tres análisis relacionales bivariados, en los que se ha asociado la situación de práctica físico-deportiva actual y anterior con la percepción de los adolescentes sobre la importancia concedida por sus padres a la práctica físico-deportiva de sus hijos, con la situación de práctica físicodeportiva del padre, así como con la de la madre. Para analizar estos pares de variables se empleó el coeficiente V de Cramer.

\section{Fase cualitativa.}

Para completar los datos del análisis cuantitativo se ha procedido a utilizar una técnica cualitativa, el grupo de discusión.

\section{Participantes}

En esta fase intervinieron los agentes educativos más cercanos a los adolescentes objeto de estudio: padres, profesores de Educación Física, profesores de otras áreas, así como los propios jóvenes, con un total de 41 participantes.

\section{Medidas}

A partir del estudio conceptual, del estado de la cuestión y de la primera lectura del contenido de los grupos de discusión llevados a cabo, se procedió a la elaboración de un mapa axiológico del ocio físico-deportivo, constituido por seis dimensiones: social, personal, saludable, estética, habilidad-forma física y lúdica. Asimismo, se definieron las categorías y subcategorías de análisis; aquí se profundiza en la subcategoría "Dimensión social de los valores en la práctica físico-deportiva" por ser la referente al objeto de estudio del presente artículo. Dicha subcategoría engloba múltiples valores, tales como la amistad, la convivencia, la cooperación, el civismo y la familia, así como algunos contravalores, caso de la competitividad insana y la obsesión por el dinero. A su vez, cada uno de estos valores y contravalores se traducen en diversas actitudes, las cuales permiten su identificación en los discursos obtenidos. La tabla 2 es reflejo de los valores, contravalores y actitudes referentes a la dimensión social. 
Tabla 2. Valores, contravalores y actitudes de la dimensión social

\begin{tabular}{|c|c|}
\hline \multicolumn{2}{|r|}{ DIMENSIÓN SOCIAL } \\
\hline VALORES & ACTITUDES \\
\hline AMISTAD & $\begin{array}{l}\text { - } \quad \text { Iniciar y mantener relaciones interpersonales. } \\
\text { - } \quad \text { Prestar ayuda desinteresada. } \\
\text { - } \quad \text { Mantener las relaciones interpersonales fuera del ámbito físico- deportivo. } \\
\text { Ponerse en el lugar del otro para la comprensión de sentimientos y situaciones. }\end{array}$ \\
\hline $\begin{array}{l}\text { DEPORTIVIDAD } \\
\text { CIVISMO }\end{array}$ & 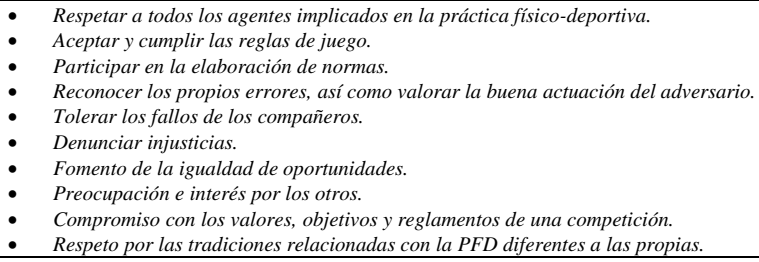 \\
\hline $\begin{array}{c}\text { COMPETITIVIDAD } \\
\text { INSANA }\end{array}$ & $\begin{array}{l}\text { - } \quad \text { Obsesión por el éxito individual en la práctica físico-deportiva. } \\
\text { - } \quad \text { Convicción de que la satisfacción de la actividad físico-deportiva está en el triunfo. } \\
\text { - } \quad \text { Pretender buenos resultados obviando el juego limpio. } \\
\text { - } \quad \text { Tendencia a la competitividad como fin exclusivo de la práctica. } \\
\text { - } \quad \text { Obsvicción de que el propio honor está ligado al éxito personal. } \\
\text { - } \quad \text { Tendencia a excluir a los menos capaces motrizmente. }\end{array}$ \\
\hline $\begin{array}{l}\text { CONVIVENCIA } \\
\text { COOPERACIÓN }\end{array}$ & $\begin{array}{ll} & \text { Trabajar de forma coordinada para conseguir metas comunes. } \\
\text { - } & \text { Buscar los beneficios del grupo antes que el beneficio personal. } \\
\text { - } & \text { Conformarse, adaptarse y aceptar las normas del grupo. } \\
\text { - } & \text { Prestar ayuda y mostrarse servicial y colaborativo sin esperar beneficio a cambio. } \\
\text { - } & \text { Fomentar la cohesión y el bienestar del grupo. } \\
\text { - } & \text { Tendencular los errores de los otros. } \\
\text { - } & \text { Mostrar agradecimiento por lo que recibe del grupo. } \\
\text { - } & \text { Convicción de la sinergia que proporciona el trabajo en equipo. } \\
\text { - } & \text { Aceptar a los demás como son. } \\
\text { - } & \text { Trabosición al diálogo. } \\
\end{array}$ \\
\hline DINERO & $\begin{array}{l}\text { - } \quad \text { Obsesión por las cuestiones económicas de la actividad físico-deportiva. } \\
\text { - } \quad \text { Interés por conseguir un buen lugar en el equipo con fines materiales. } \\
\text { - } \quad \text { Considerar el dinero como hándicap para el desarrollo de la PFD. } \\
\text { - Convicción de que el dinero es un poderoso condicionante en la práctica PFD. } \\
\text { - Considerar que las cuestiones económicas son el fin de la práctica físico-deportiva. }\end{array}$ \\
\hline FAMILIA & $\begin{array}{l}\text { - } \quad \text { Reconocer que su práctica físico-deportiva es fruto de su educación familiar. } \\
\text { - } \quad \text { Creencia de que sus patrones de AFD responden a la tradición familiar. } \\
\text { - } \quad \text { Disposición a reproducir modelos familiares. } \\
\text { - } \quad \text { Propensión al respeto de la tradición deportiva. } \\
\text { - Disposición al aprendizaje y práctica de eventos físico-deportivos familiares. }\end{array}$ \\
\hline
\end{tabular}

Esta dimensión estima la esfera social de los valores en el ocio físicodeportivo como condicionante de dicha práctica, en la que pueden establecerse cuestiones referentes a las relaciones de grupo y/o amistosas derivadas de dichas experiencias, sus posibilidades para la socialización y la convivencia, así como los posibles conflictos emanados de las mismas, entre otras. Así pues, este factor se constituye como elemento que puede colaborar en gran medida en el bienestar del individuo, siendo definitivo para la calidad de vida y la riqueza social de la persona.

Además, esta subcategoría se agrupa en dos apartados; el primero, referente a los agentes sociales implicados en la práctica físico-deportiva del adolescente y, el segundo, concerniente a los condicionantes sociales de dichas experiencias. 
El primer apartado se constituye en nuestro objeto de estudio y engloba argumentos que aluden a la percepción de que unos padres activos físicamente influyen positivamente en el inicio y mantenimiento de la práctica de sus hijos, o de que una valoración familiar desfavorable hacia la práctica condiciona negativamente el hábito en el adolescente, o la opinión de que los padres utilizan las experiencias de OFD con el objetivo de mantener "ocupados" a sus hijos e, incluso, la estimación de que los padres favorecen el abandono de la práctica a medida que los niños se hacen mayores, cuando éstos requieren más tiempo y esfuerzo para dedicar a las obligaciones académicas.

A modo de ejemplo, se muestran disertaciones emanadas de los colectivos, objeto de estudio, relacionadas con nuestro ámbito de trabajo.

Aunque ni su padre ni yo hacemos deporte, esa sensación del grupito del equipo, de sus amigos y sus amigas, eso les arrastra un montón a hacer deporte. La convivencia con el equipo y, después de ir a entrenar, estar un poco en el patio les influye mucho para hacer deporte.

Creo que buscan pasárselo bien y conocer más, abrirse en amistades (...) cuando haces deporte abres mucho más el campo de amistades.

Hoy en día hay muchos padres que al deporte no le dan tanta importancia, porque creen que su hijo tiene que estar más preparado que el resto de la media para poder encontrar un mejor trabajo y para sacar unas mejores notas. Y llevan a los chavales a otros campos: las nuevas tecnologías, mecanografía, Internet, idiomas, etc. Y eso también, a estos niveles, está pasando en la adolescencia.

(Colectivo de Padres)

A muchas personas les influye el hecho de que para los padres los estudios son lo primero y si te ven que vas mal, pues te van a quitar el fútbol.

La influencia de la familia, para mí, es toda.

A mí me interesa hacer deporte, pero me han dicho mis padres que primero son los estudios y más adelante, si ven que van bien y si me apetece hacer algún deporte, que lo haga. Pero de momento que no.

(Colectivo de Alumnos)

Los padres son fundamentales, pero por más que les explicas que se puede estudiar y hacer deporte a la vez, hay muchos que se cierran.

Los chicos buscan relacionarse con sus compañeros y formar parte de un grupo.

La PFD les enseña a convivir. Una serie de cosas fundamentales.

(Colectivo de Profesores de otras áreas)

Llega un momento en que los padres dicen: "Oye, como tú en este deporte no haces más que perder el tiempo, dedicate a estudiar, no hagas actividad fisica". El deporte es una forma de integración.

Cuando son pequeños a los padres no nos importa pagar 60 euros para que hagan deporte y el niño esté entretenido y no nos dé la lata.

(Colectivo de Profesores de Educación Física)

Las categorías de análisis se validaron a través del juicio de expertos, los cuales identificaron las unidades textuales de uno de los grupos de discusión (profesores de Educación Física), registrándolas en las categorías y subcategorías consideradas. Los datos obtenidos se transcriben al SPSS, 14.0 y se aplica la fórmula Kappa de Cohen (Cohen, 1960, 1968) para medir la fiabilidad entre evaluadores, obteniéndose unas 
medidas de acuerdo positivas, de lo que resulta un proceso de validación satisfactorio. La figura 1 es reflejo de los resultados obtenidos en referencia a la subcategoría analizada.

Figura 1. Dimensión social de los valores

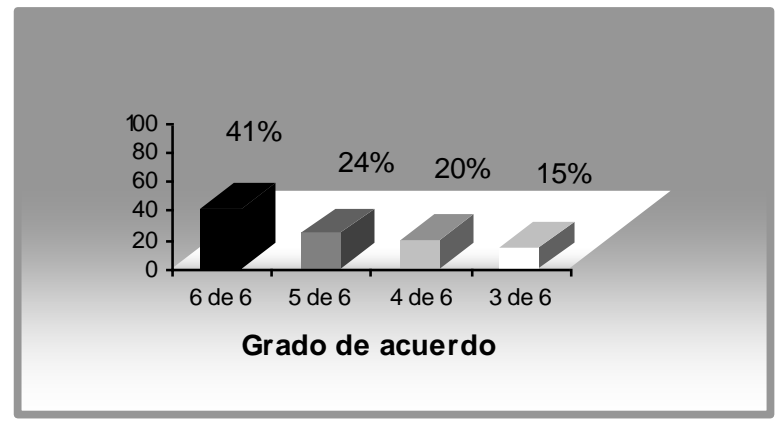

Los coeficientes Kappa obtenidos entre el investigador principal y cada uno de los expertos, así como el grado de acuerdo estimado en función del índice Kappa, según los márgenes propuestos por Landis y Koch (1977), reflejan medidas de acuerdo favorables.

Respecto al grado de concordancia entre el resto de expertos, las puntuaciones en ningún momento han sido inferiores a 0.5 . Son índices que reflejan un grado de acuerdo entre moderado, bueno y muy bueno.

A la luz de los resultados obtenidos puede darse por satisfactorio el proceso de validación de categorías de la presente investigación.

Finalmente, se desciende a una nueva exploración del contenido de los grupos de discusión, volviéndose a registrar aquellos correspondientes a la dimensión que nos ocupa con el fin de perfeccionar dicho análisis, así como los resultados y su interpretación. Una vez identificadas estas unidades textuales se procede a la lectura pormenorizada de las mismas, así como al análisis en profundidad de cada uno de los relatos obtenidos.

\section{Procedimiento}

Se realizaron cuatro grupos de discusión, uno para cada uno de los agentes educativos más cercanos: Padres y madres, profesores de Educación Física, profesores del resto de áreas y estudiantes de Educación Secundaria Obligatoria. La selección de los participantes fue estructural, eligiendo la formación de los distintos colectivos según criterios de pertenencia. Se tuvieron en cuenta unos mínimos de homogeneidad -pertenencia a cada colectivo- y heterogeneidad, respetándose un reparto equitativo en función del sexo, tipo de centro y ubicación geográfica (González, 1997; Suárez, 2005). Así pues, los 4 grupos de discusión estuvieron configurados por 8 padres y madres de alumnos de Secundaria, 10 profesores de Educación Física, 8 profesores del resto de áreas y 15 estudiantes. 
Los relatos surgidos en estos grupos de discusión fueron grabados y transcritos en formato RTF y se procede a un análisis de los mismos con el apoyo del paquete informático NUD*IST. Cada unidad textual hace alusión a una frase con sentido y significado que puede ser identificada en una de las categorías o subcategorías de análisis.

\section{RESULTADOS}

En referencia a la importancia concedida al ámbito del ocio físico-deportivo como espacio susceptible de favorecer valores que optimizan la convivencia saludable, se debe apuntar que los resultados procedentes del cuestionario sitúan en quinto lugar los beneficios sociales obtenidos en la práctica físico-deportiva (Figura 2), según la percepción de los alumnos, con un $12.6 \%$ de los casos. En dicho aspecto social se hallan cuestiones como conocer nuevos amigos, socializarse y relacionarse, así como disfrutar con otras personas y cooperar con los demás.

Figura 2. Beneficios físico-deportivos

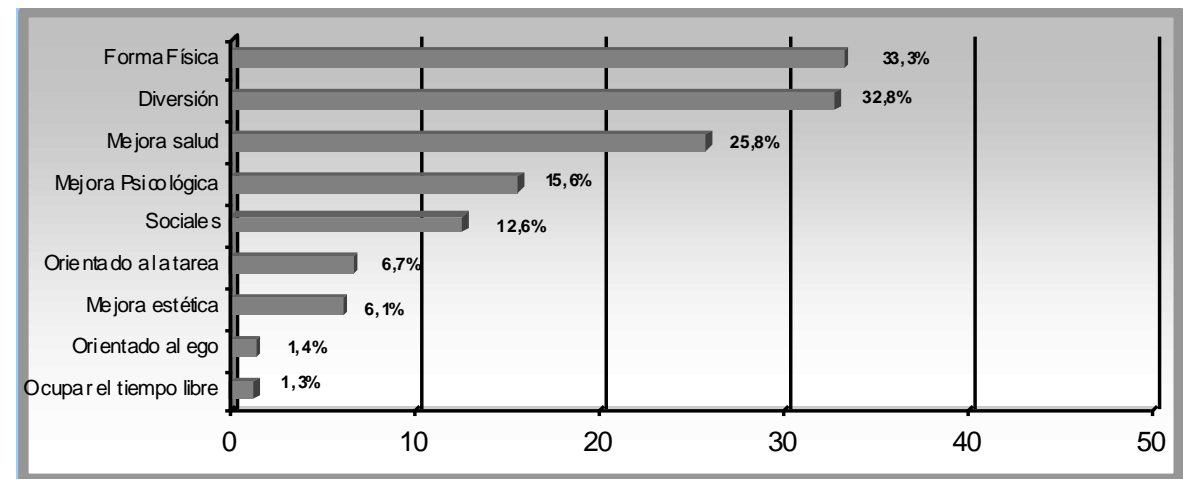

En la misma línea, respecto a la fase cualitativa, se obtienen 547 unidades textuales concernientes a las dimensiones de los valores en la práctica físico-deportiva, de las que el $80 \%$ se refieren a la dimensión social de los mismos, reflejándose una importante cuantía de discursos que ponen de manifiesto, tal y como se expresó en el apartado de metodología, que las experiencias de ocio físico-deportivo bien encauzadas pedagógicamente se constituyen en el terreno propicio para el fomento de valores que optimizan la adecuada socialización del adolescente y, consecuentemente, educan en una convivencia saludable.

Asimismo, como refleja la figura 3 , un $29 \%$ de las unidades textuales registradas alusivas a la dimensión social del mapa axiológico corresponden al colectivo de padres, cuestión que refleja la importancia concedida por los progenitores a los valores para la socialización y la convivencia. 
Figura 3. Dimensión Social según colectivos

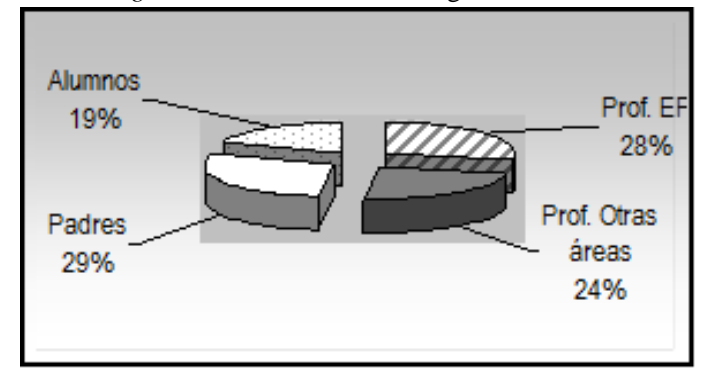

Por otro lado, al indagar en la influencia familiar sobre la situación de práctica de los adolescentes, la mayoría de éstos, un $78.2 \%$, percibe que sus padres le conceden una importancia adecuada a su ocio físico-deportivo. Incluso, al analizar el estado de la práctica de los adolescentes, puede constatarse que la situación de práctica actual y anterior que presentan los jóvenes está estrechamente vinculada con el nivel de importancia que los padres conceden a la práctica físico-deportiva $\left(0.213^{* * *}\right)$, no así con el comportamiento físico-deportivo manifestado por los progenitores.

En este estudio queda verificado que cuando los padres conceden un valor importante a la AFD es significativamente superior la tasa de adolescentes que practica habitualmente frente a la de aquellos que nunca han realizado actividad física (no practico ni practicaba $=13.2 \%$ y practica y practicaba $=54 \%$, intervalo: $-15.08 ;-62.51$, así como a la de aquellos adolescentes que han abandonado su práctica (no practico pero practicaba $=21.3 \%$ y practica y practicaba $=54 \%$, intervalo: $-22.96 ;-42.43$ ). La tabla 3 refleja el análisis inferencial de contrastes entre la situación de práctica de los jóvenes y la importancia otorgada por los progenitores a la AFD.

Tabla 3. Análisis inferencial de contrastes entre la situación de práctica y la importancia concedida por los padres a la AFD

\begin{tabular}{|c|c|c|c|c|c|c|c|c|c|c|}
\hline \multirow{2}{*}{$\begin{array}{l}\text { IMPORTANCIA } \\
\text { CONCEDIDA } \\
\text { POR LOS } \\
\text { PADRES A LA } \\
\text { AFD }\end{array}$} & \multicolumn{4}{|c|}{$\begin{array}{l}\text { SITUACIÓN DE PRÁCTICA } \\
\text { ACTUAL Y ANTERIOR }\end{array}$} & \multirow{2}{*}{$\begin{array}{c}\text { Intervalo } \\
\text { entre } \\
\text { nunca he } \\
\text { practicado } \\
\text { y no } \\
\text { practico } \\
\text { pero antes } \\
\text { practicaba }\end{array}$} & \multirow{2}{*}{$\begin{array}{c}\text { Intervalo } \\
\text { entre } \\
\text { nunca he } \\
\text { practicado } \\
\text { y practico } \\
\text { pero antes } \\
\text { no } \\
\text { practicaba }\end{array}$} & \multirow{2}{*}{$\begin{array}{c}\text { Intervalo } \\
\text { entre } \\
\text { nunca he } \\
\text { practicado } \\
\text { y práctico } \\
\text { y } \\
\text { practicaba }\end{array}$} & \multirow{2}{*}{$\begin{array}{c}\text { Intervalo } \\
\text { entre no } \\
\text { practico } \\
\text { pero antes } \\
\text { practicaba } \\
\text { y practico } \\
\text { pero antes } \\
\text { no } \\
\text { practicaba }\end{array}$} & \multirow{2}{*}{$\begin{array}{c}\text { Intervalo } \\
\text { entre no } \\
\text { practico } \\
\text { pero antes } \\
\text { practicaba } \\
\text { y práctico } \\
\text { y } \\
\text { practicaba }\end{array}$} & \multirow{2}{*}{$\begin{array}{c}\text { Intervalo } \\
\text { entre } \\
\text { practico } \\
\text { pero antes } \\
\text { no } \\
\text { practicaba } \\
\text { y práctico } \\
\text { y } \\
\text { practicaba }\end{array}$} \\
\hline & 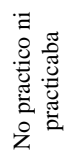 & 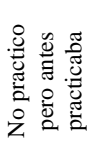 & 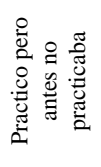 & 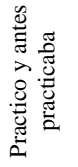 & & & & & & \\
\hline Nada o poca & $47.6 \%$ & $39.4 \%$ & $19.6 \%$ & $11.4 \%$ & $\begin{array}{r}28.05 \\
-11.65\end{array}$ & $\begin{array}{c}53.17 \\
2.52\end{array}$ & $\begin{array}{l}55.00 \\
17.39\end{array}$ & $\begin{array}{c}39.35 \\
0.24\end{array}$ & $\begin{array}{l}37.33 \\
18.66\end{array}$ & $\begin{array}{c}26.69 \\
-10.29\end{array}$ \\
\hline Normal & $32.8 \%$ & $33.1 \%$ & $36.1 \%$ & $31.5 \%$ & $\begin{array}{r}21.91 \\
-22.51 \\
\end{array}$ & $\begin{array}{c}22.71 \\
-29.31 \\
\end{array}$ & $\begin{array}{c}22.31 \\
-19.71 \\
\end{array}$ & $\begin{array}{r}14.98 \\
-20.98 \\
\end{array}$ & $\begin{array}{l}11.00 \\
-7.80 \\
\end{array}$ & $\begin{array}{r}21.07 \\
-11.87\end{array}$ \\
\hline $\begin{array}{l}\text { Bastante o } \\
\text { mucha }\end{array}$ & $13.2 \%$ & $21.3 \%$ & $42.3 \%$ & $54 \%$ & $\begin{array}{r}17.05 \\
-33.25\end{array}$ & $\begin{array}{l}-1.19 \\
-57.00\end{array}$ & $\begin{array}{l}-15.08 \\
-62.51\end{array}$ & $\begin{array}{l}-3.35 \\
-38.64\end{array}$ & $\begin{array}{l}-22.96 \\
-42.43\end{array}$ & $\begin{array}{c}3.81 \\
-27.21\end{array}$ \\
\hline
\end{tabular}

Asimismo, es interesante comprobar que los motivos de inicio a la práctica físico-deportiva, según la percepción de los adolescentes, se fundamentan en razones 
familiares y sociales, cuestiones destacadas en segundo y tercer lugar, respectivamente. Reflejo de ello es la figura 4.

Figura 4. Motivos de inicio en la PFD

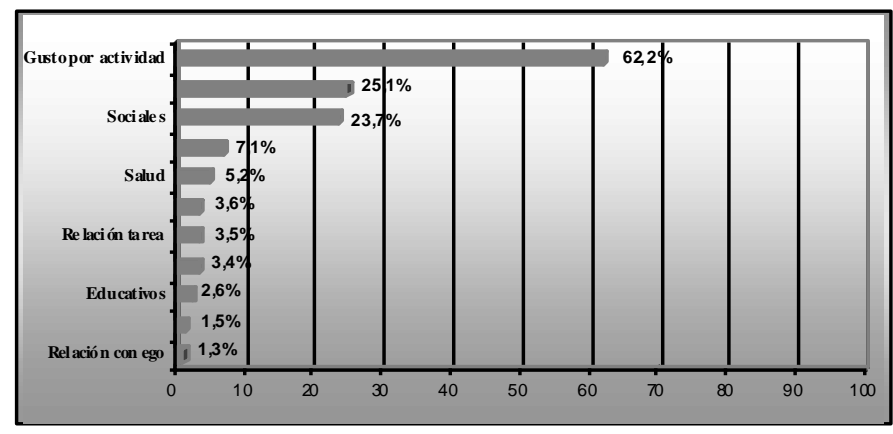

En esta línea, aludiendo a los resultados obtenidos en la fase cualitativa, se hace preciso poner de manifiesto que en el apartado I de la subcategoría "Dimensión social de los valores" -denominado "agentes sociales", que acopia el 49\% de los testimonios-, un $11 \%$ de los mismos hacen referencia a la familia como factor condicionante de la práctica físico-deportiva (Figura 5).

\section{Figura 5. Apartado I dimensión social}

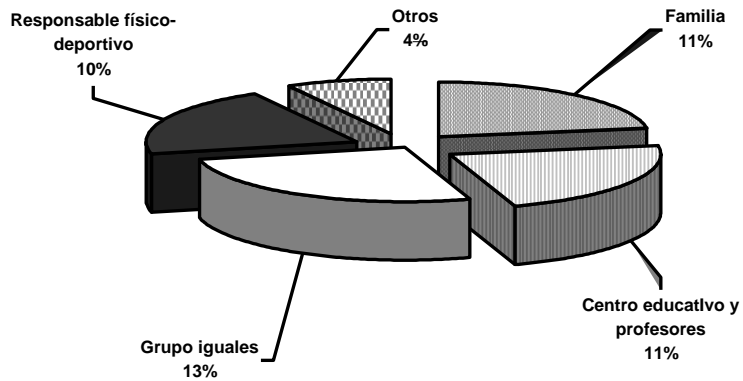

Figura 6. Familia por colectivos

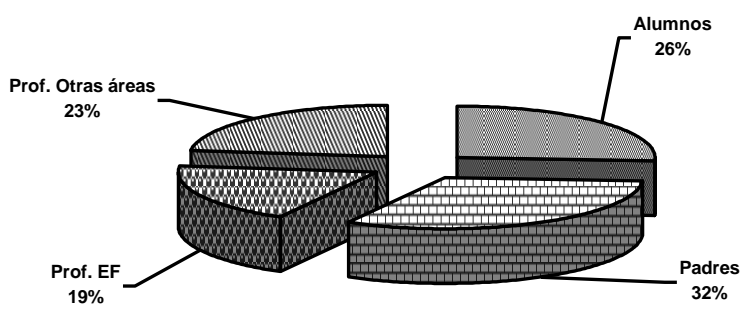


Por último, la figura 6 pone de manifiesto la distribución porcentual por colectivos de los testimonios referentes a aquellos factores familiares que se constituyen en condicionantes de la práctica físico-deportiva del adolescente. Se observa que aunque son los padres quienes soportan el mayor porcentaje de registros sobre este subapartado (32\%), el resto de colectivos realiza un importante número de testimonios al respecto que merecen consideración.

\section{DISCUSIÓN Y CONCLUSIONES}

La dimensión social del mapa axiológico del OFD ha sido la subcategoría con mayor cuantía de discursos, descubriéndose actitudes que ponen de manifiesto que tanto los padres como los profesores, así como los propios jóvenes conceden gran importancia a la amistad, la socialización, la cooperación, el sentimiento de pertenencia al grupo y la convivencia como valores sociales obtenidos de la participación en experiencias de ocio físico-deportivo. Además, consideran que dicha práctica se constituye en un contexto propicio para el fomento de los valores sociales mencionados.

No obstante, cabe resaltar que nuestros colectivos objeto de análisis ponen en evidencia ciertos contravalores, caso de la competitividad insana, la obcecación por el triunfo a cualquier precio y la obsesión por el dinero que, según su percepción, emanan de la práctica físico-deportiva en más ocasiones de las deseadas.

Esta realidad no hace sino ratificar que el OFD es, a priori, un contexto axiológico neutro, que posee el potencial de fomentar tanto valores como antivalores, dependiendo del enfoque pedagógico que se lleve a cabo (Borrás, 2004, GarcíaFerrando, 1990, Giebink y McKencie, 1985 y Lee y Cook, 1990).

Siendo conscientes de las limitaciones de nuestro estudio, se vaticina que no son los padres, en el estudio que nos ocupa, los responsables de que germinen los antivalores mencionados, dado que hemos constatado que éstos respaldan una práctica promotora de valores como el esfuerzo, el disfrute, la convivencia y la socialización, así como que prevenga la aparición de contravalores, tales como la competitividad insana o los comportamientos obsesivos.

No obstante, como se observa con posterioridad, se han detectado discrepancias perceptivas entre padres, por un lado, y profesores y jóvenes, por otro, acerca del poder de influencia de la familia en el ocio físico-deportivo de sus hijos.

Se considera que se debe seguir profundizando en el análisis de los contravalores detectados, con el fin indagar en el origen de los mismos y facilitar el establecimiento de aquellas líneas pedagógicas de actuación que optimicen la educación axiológica en el ámbito que nos ocupa, previniendo en lo posible la aparición de antivalores.

Con todo ello y ante esta actitud positiva de los progenitores, se estima esencial favorecer la creación de escuelas de padres en las que se les ofrezcan estrategias de intervención encaminadas al fomento de valores que optimicen la educación para una convivencia saludable, destacándose el respeto como valor cardinal. 
Por otro lado, se ha constatado que los adolescentes destacan aquellos aspectos relacionados con la amistad, las relaciones sociales y la convivencia con sus amigos como motivo para la participación en las experiencias de ocio físico-deportivo. A pesar de que diferentes autores ya han obtenido conclusiones similares (Castillo y Balaguer, 2001; Griffin, 1985; Moreno y Hellín, 2002; Ponce de León et al., 2009; Velázquez y Hernández, 2003), la presente investigación complementa lo anterior constatando que, además de los adolescentes, son los padres y los profesores quienes conceden idéntica categoría a estos valores como impulsores de dicho hábito, por lo que se debería utilizar la fuerza de esta percepción común para convertir este ámbito de educación no formal en un trampolín que satisfaga estas necesidades sociales y, por ende, optimice el desarrollo integral del adolescente.

Asimismo, nuestra investigación corrobora el papel fundamental de estas experiencias en el fomento de la integración social, así como de las habilidades y competencias sociales, además de constituirse en una herramienta fundamental para prevenir el aislamiento social fortaleciéndose, de este modo, algunas conclusiones expresadas por otros autores (Pelegrín, 2004; Ponce de León et al., 2009; Raga, 2002; Reitman et al., 2005).

Se considera que todas estas cuestiones deben tenerse en alta consideración en las propuestas de intervención pedagógica en este ámbito para favorecer tanto la adhesión y afianzamiento de este estilo de vida como su aprovechamiento para la mejora de la socialización del adolescente y la prevención de situaciones de exclusión personal y social.

En otro orden de cosas, es de destacar como conclusión de la presente investigación que tanto la importancia que el propio adolescente concede a la práctica físico-deportiva como el comportamiento físico-deportivo demostrado por el mismo se constituyen en aspectos que fundamentan su cultura físico-deportiva; ambas cuestiones se enraízan en la importancia que confieren los padres a este tipo de prácticas. Esta afirmación defiende que la familia es un factor con alto poder condicionante en las experiencias de ocio físico-deportivo del adolescente (Hassandra et al., 2002; Moreno y Cervelló, 2004; Nuviala et al., 2003; Vilhjalmsson y Thorlindsson, 1998).

En nuestra investigación dicha influencia se sustenta en la importancia que otorgan los padres a dicha práctica en la adquisición y mantenimiento de los hábitos físico-deportivos de sus hijos, así como en la consideración de que este ámbito es fuente de fomento de hábitos saludables.

Sin embargo debe resaltarse, como aportación del presente estudio, que la práctica físico-deportiva de los padres no se constituye en un aspecto determinante del que bebe el comportamiento físico-deportivo del joven ni condiciona el valor que éste concede a dichas prácticas. No obstante, en un estudio con una población similar y cercana a la del nuestro, Nuviala et al. (2003), se llega a la conclusión de que la práctica física de los padres está asociada con la práctica del hijo. Ahora bien, la población objeto de este estudio estuvo formada por escolares de Primaria y Secundaria, resaltando, no obstante, que entre los alumnos de Primaria la influencia de los padres es mayor que entre los adolescentes de Secundaria. Este hecho va en consonancia con la afirmación de Hendry, Shucksmith, Love y Glendinning (1993), quienes consideran que los 
adolescentes tienen una menor dependencia de los padres, a favor de los amigos, a la hora de realizar actividad físico-deportiva.

En otro sentido, se considera fundamental destacar, de la presente investigación, la discordancia perceptiva manifestada entre los padres, por un lado, y el resto de colectivos, por otro, en referencia al poder condicionante de los progenitores sobre el OFD de sus hijos. Tanto los jóvenes como los docentes recriminan a los padres el hecho de conceder excesiva prioridad a las cuestiones académicas en detrimento del ámbito físico-deportivo. Además, los profesores reprochan ciertas actitudes puestas de manifiesto por algunos padres y madres que utilizan dicha práctica con propósitos más dirigidos al beneficio propio que al de sus hijos. Paradójicamente, los padres declaran estar siempre dispuestos a sacrificar su tiempo y su esfuerzo si prevén que es para algo que va a reportar a sus hijos ventajas en su desarrollo como personas.

Son cuestiones que reclaman la necesidad de continuar profundizando en estas discrepancias perceptivas, dado que se intuye que los padres no son realmente conscientes del enorme poder de influencia que ejercen sobre sus hijos en el inicio y mantenimiento del hábito físico-deportivo o, en su defecto, en la no práctica y el abandono del mismo.

Conscientes de las restricciones de nuestro estudio, se manifiesta la necesidad de profundizar en las líneas abiertas en la presente investigación a través de un análisis multivariable que nos ofrezca la causa-efecto de un determinado comportamiento físicodeportivo. Los potenciales resultados permitirían el diseño de propuestas de intervención educativa que optimicen la educación en valores en los ámbitos no formales, en general $\mathrm{y}$, en el contexto del ocio físico-deportivo, en particular.

\section{Agradecimientos}

Agradecemos a la Consejería de Educación, Cultura y Deporte del Gobierno de La Rioja el soporte financiero concedido al estudio dentro del Plan Riojano de I+D+i (Ref. Fomenta 02/2007).

\section{REFERENCIAS}

Borrás, P.A. (2004). Intervención para la promoción de la deportividad en el fútbol cadete en Mallorca. Tesis doctoral. Facultad de Educación. Universidad de las Islas Baleares. Islas Baleares. España.

Castillo, I. y Balaguer, I. (2001). Dimensiones de los motivos de práctica deportiva de los adolescentes valencianos escolarizados. Apunts: Educación Física y Deportes, 63, 22-29.

Cohen, J.A. (1960). Coefficient of agreement for nominal scales. Educational and Psychological Measurement, 20, 37-46.

Cohen, J.A. (1968). Weighted kappa: Nominal scale agreement with provision for scaled disagreement of partial credit. Psychological Bulletin, 70, 213-220.

Delors, J. (1996). La educación encierra un tesoro. Madrid: Santillana. Ediciones UNESCO.

Edo, J.A. (2004). Adolescencia y deporte en el Principado de Andorra al inicio del siglo XXI. Perspectiva desde una triangulación de fuentes de información. Apunts. Educación Física y Deportes, 77(3), 5-11.

García-Ferrando, M. (1990). Aspectos sociales del deporte. Madrid: Alianza Deporte. 
Giebink, M.P. y McKencie, T.L. (1985). Teaching sportmanship in physical education and recreation: an analysis of interventions and generalization effects. Journal of Teaching in Physical Education, 4, 167-177.

Griffin, P. (1985). Boys' participation styles in a middle school physical education team sports unit. Journal of Teaching in Physical Education, 4, 100-110.

Hassandra, M., Goudas, M. y Chroni, S. (2002). Examining factors associated with intrinsic motivation in physical education: a qualitative approach. Psychology of Sport and Exercise, 4, 211-223.

Hendry, L.B., Shucksmith, J., Love, J.G. y Glendinning, A. (1993). Young people's leisure and lifestyles. London: Routledge.

Jiménez, C. (2005). Convivencia: conceptualización y sugerencias para la praxis. Puntos de vista, $1,7-31$.

Kant, I. (1983). Pedagogía. Madrid: Akal.

Kremer-Sadlik, T. y Kim, de J.L. (2007). Lessons from sports: Children's socialization to values through family interaction during sports activities. Discourse and Society, 18(1), 35-52.

Landis, J.R. y Koch, G. (1977). The measurement of observer agreement for categorical data. Biometrics, 33, 159-174.

Lee, M.J. y Cook, C. (1990). Review of the literature on fairplay with special reference to children's sport. Estrasburgo: Council of Europe.

López-Pastor, V.M. (2003). La evaluación compartida en educación física. En Actas del III Congreso Estatal de Actividades Físicas Cooperativas. Valladolid: La Peonza.

Marina, J.A. (2006). Aprender a Convivir. Barcelona: Ariel.

Moreno, J.A. y Cervelló, E. (2004). Pensamiento del alumno hacia la educación física: su relación con la práctica deportiva y el carácter del educador. Enseñanza, 21, 345-362.

Moreno, J.A. y Hellín, G. (2002). ¿Es importante la Educación Física? Su valoración según la edad del alumno y el tipo de centro. Revista Internacional de Medicina y Ciencias de la Actividad Física y del Deporte, 8. Recuperado el 5 de diciembre de 2007 de: http://cdeporte.rediris.es/revista/.

Nuviala, A., Ruiz-Juan, F. y García-Montes, M.E. (2003). Tiempo libre, ocio y actividad física en los adolescentes. La influencia de los padres. Retos. Nuevas tendencias en Educación Física, Deporte y Recreación, 6, 13-20.

Pelegrín, A. (2004). El comportamiento agresivo y violento. Factores de riesgo y protección como mediadores de inadaptaciones y adaptaciones en la socialización del niño y el adolescente. Murcia: Universidad de Murcia. [Tesis Doctoral]

Ponce de León, A., Sanz, E., Ramos, R. y Valdemoros, M. (2010). Cuestionario de motivaciones, actitudes y comportamientos en el ocio físico-deportivo juvenil. MACOFYD. Logroño: Universidad de La Rioja.

Ponce de León, A., Sanz, E., Valdemoros, M.A. y Ramos, R. (2009). Los valores personales en el ocio físico-deportivo. Un estudio con jóvenes, padres y profesores. Bordón, 61(1), 29-41.

Raga, J.L. (2002). Influencia de la actividad física y el deporte en el desarrollo de la competencia social de los adolescentes. Tesis doctoral. Facultad de Educación. Universidad de Oviedo. Oviedo. España.

Ramos, R., Sanz, E., Ponce de León, A. y Valdemoros, M.A. (2009). La percepción del ocio saludable en la práctica físico-deportiva juvenil. Un análisis cualitativo. Revista Apunts: Educación Física y Deportes, 95, 24-31.

Reitman, D., O'Callaghan, P.M. y Mitchell, P. (2005). Parent as coach: Enhancing sports participation and social behaviour for ADHD-diagnosed children. Child and Family Behavior Therapy, 27(2), 57-68. 
Suárez, F.A. (2005). El grupo de discusión. Una herramienta para la investigación cualitativa. Barcelona: Alertes.

Valdemoros, M.A. (2010). Los valores en el ocio físico-deportivo. Análisis y propuestas educativas. Logroño: Universidad de La Rioja.

Velázquez, R. y Hernández, J.L. (2003). Deporte y formación de actitudes y valores en los niños, niñas y adolescentes de la comunidad de Madrid. Revista de Educación, 331, 369-398.

Vilhjalmsson, R. y Thorlindsson, T. (1998). Factors related to physical activity: a study of adolescents. Social Science and Medicine, 47(5), 665-675.

Vizcarra, M.T., Macazaga, A.M. y Rekalde, I. (2006). ¿Con qué deporte escolar sueñan las familias? Apunts. Educación Física y Deportes, 86, 97-107.

Yuste, N. y Pérez, M.C. (2008). Las cuestiones familiares como causa de la violencia escolar según los padres. European Journal of Education and Psychology, 2(1), 19-27.

Recibido: 1 de marzo de 2010 Recibido modificaciones: 22 de noviembre de 2010

Aceptado: 26 de noviembre de 2010 